\title{
Spin-stand imaging of overwritten data and its comparison with magnetic force microscopy
}

I. D. Mayergoyz ${ }^{\text {a) }}$ and C. Tse

Electrical and Computer Engineering Department, University of Maryland, College Park, Maryland 20742

C. Krafft

Laboratory for Physical Sciences, College Park, Maryland 20740

R. D. Gomez

Electrical and Computer Engineering Department, University of Maryland, College Park, Maryland 20742

A new technique of magnetic imaging on a spin-stand [Mayergoyz et al., J. Appl. Phys. 87, 6824 (2000)] is further developed and extensively tested. The results of successful imaging of digital patterns overwritten with misregistration ranging from 0.3 to $0.07 \mu \mathrm{m}$ are reported. The results are compared with magnetic force microscopy (MFM) images and the conclusion is reached that the spin-stand imaging technique can provide (at least) the same level of resolution and accuracy as the MFM imaging technique. (C) 2001 American Institute of Physics. [DOI: 10.1063/1.1359233]

It has long been recognized that the imaging of magnetization patterns recorded on hard drive disks is a source of valuable information that may enhance our understanding of recording processes and assist in the design of new recording systems. The magnetization imaging is routinely performed by using magnetic force microscopy (MFM) ${ }^{1}$ It has been realized that MFM has the following intrinsic limitations: (1) low rate of image acquisition, (2) special requirements for the preparation of the sample to be imaged, and (3) virtual impracticality of fast accumulation of numerous images of the same target area in order to increase the signal-to-noise ratio.

Recently, a new technique of magnetic imaging on a spin-stand has been developed. ${ }^{2}$ In this technique, raw image acquisition is performed by scanning a target area of a hard drive disk by a magnetoresistive (MR) head in the along- and cross-track directions. Scanning in the along-track direction is realized due to the rotation of the disk, while scanning in the cross-track direction is achieved by using very small and accurately controlled radial displacements of the head. As a result of this scanning mechanism, the spin-stand imaging technique has the following advantages over conventional MFM imaging: a high rate of image acquisition, increase in the signal-to-noise ratio due to multiple imaging of the same target area, and performance of imaging under similar conditions as in conventional hard disk drives. However, due to the nonlocalized nature of the magnetoresistive head in the cross-track direction, the collected images can be quite distorted. In addition, the collected images are scalar in nature, while magnetization distributions are vector fields. For these reasons, the collected images must be treated as raw images, and image reconstruction is needed in order to retrieve the actual magnetization distributions from the raw images. The image reconstruction technique is based on the response function characterization of the MR reading element and can be described as follows.

${ }^{a)}$ Electronic mail: isaak@eng.umd.edu
The position of the scanning MR element can be identified by the $x$ and $y$ coordinates of its center. The recorded magnetization distribution can be characterized by the equivalent distribution of virtual magnetic charges $\sigma_{m}\left(x^{\prime}, y^{\prime}\right)$ :

$$
\sigma_{m}\left(x^{\prime}, y^{\prime}\right)=-\mu_{0} h \operatorname{div} \mathbf{M},
$$

where $h$ is the thickness of the recording media and it is tacitly assumed that the recorded magnetization is uniform over the media thickness and, for this reason, $\operatorname{div} \mathbf{M}$ has the meaning of "surface" divergence.

The distribution of virtual magnetic charges is equivalent in the sense that they create the same magnetic field as the actual magnetization distribution. This magnetic field causes the signal collected by the MR element. This signal can be viewed as the superposition of the signals due to the elementary magnetic charges distributed over the disk surface. The last assertion can be mathematically expressed as follows:

$$
S(x, y)=\iint R\left(x-x^{\prime}, y-y^{\prime}\right) \sigma_{m}\left(x^{\prime}, y^{\prime}\right) d x^{\prime} d y^{\prime} .
$$

Here $S(x, y)$ is the signal of the MR element, while $R\left(x-x^{\prime}, y-y^{\prime}\right)$ can be interpreted as the response function of the MR element. This function has the physical meaning of the signal induced in the MR element at position $(x, y)$ by the point unit magnetic charge located at position $\left(x^{\prime}, y^{\prime}\right)$.

In order to experimentally determine the response function, an isolated sharp transition is first written. This transition is then trimmed by using dc erasure on both sides of the same track. As a result, a "tiny" isolated spot of magnetic charges is written that can be viewed as an approximation to a point charge. The MR reading element can now be used to measure the signal as a function of relative position with respect to the recorded "point" magnetic charge. This signal can be interpreted as a scaled version of $R\left(x-x^{\prime}, y-y^{\prime}\right)$. An example of the spin-stand measurement of the response function of the MR element is shown in the left plot of Fig. 1. To get the information about geometric dimensions of the 

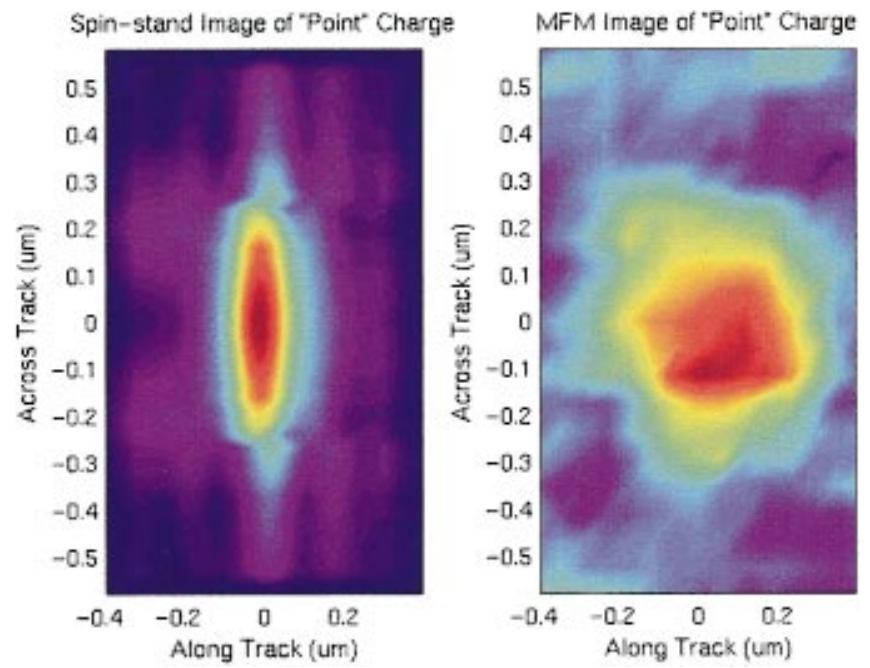

FIG. 1. (Color) Spin-stand image of a head response function of a MR element (left plot) and a MFM image of a tiny spot of magnetic charges (right plot).

recorded tiny spots of magnetic charges, the magnetic force microscope was used. An example of the MFM image of the "point" magnetic charge is shown in the right plot of Fig. 1. It is worthwhile to mention that we were able to record and measure the response function for such tiny spots of magnetic charges that their counterpart MFM images were found to be elusive and could not be clearly observed.

Having determined the response function, formula (2) can be viewed as a convolution integral equation that relates the raw image $S(x, y)$ to the distribution of virtual magnetic charges $\sigma_{m}\left(x^{\prime}, y^{\prime}\right)$ which in turn is related to the $\operatorname{div} \mathbf{M}$ through Eq. (1). There is no way to reconstruct the actual vectorial field of recorded magnetization by using only for-
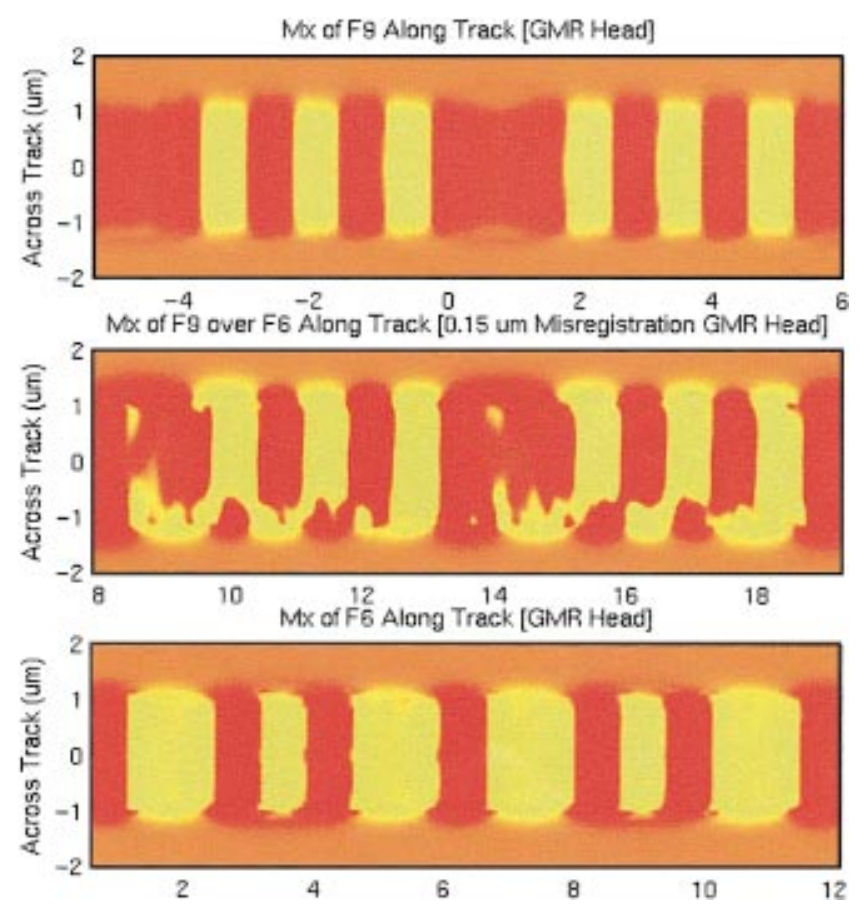

FIG. 2. (Color) Reconstructed image of F6 overwritten by F9: $0.15 \mu \mathrm{m}$ misregistration.
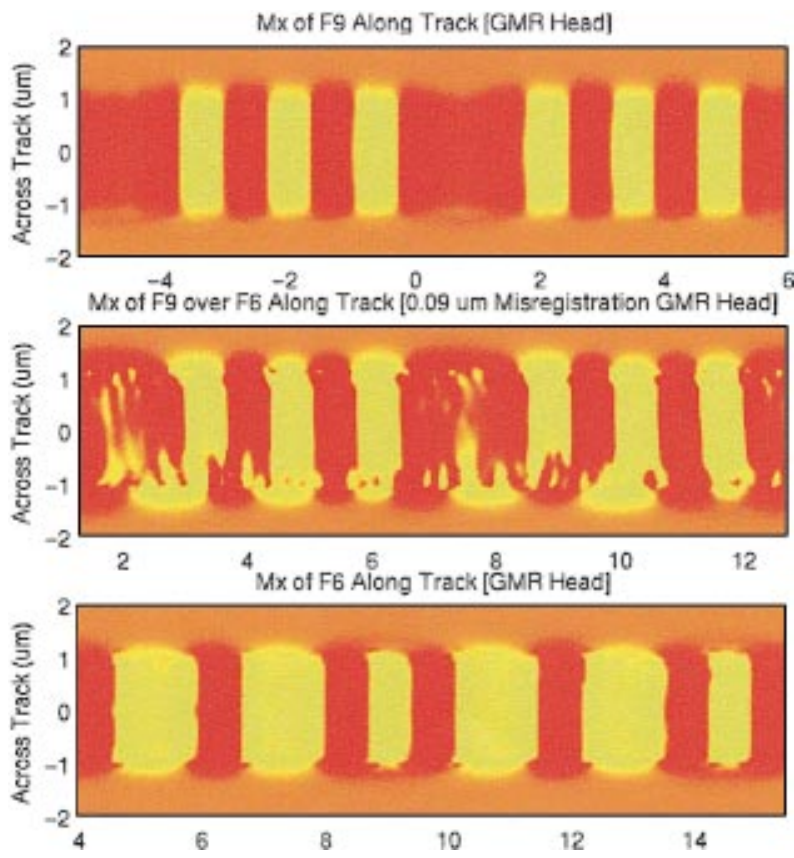

FIG. 3. (Color) Reconstructed image of F6 overwritten by F9: $0.09 \mu \mathrm{m}$ misregistration.

mulas (1) and (2). To circumvent this difficulty, we have used the known fact ${ }^{3-5}$ that only the curl-free component of magnetization can be retrieved from MR measurements. This is because the curl-free component of magnetization distribution is the field producing part of the total magnetization distribution. For this reason, only this component is sensed by the MR element. The last statement can be best illustrated by an example of a dc erased track. In this example, there exists nonzero magnetization within the track, however, this
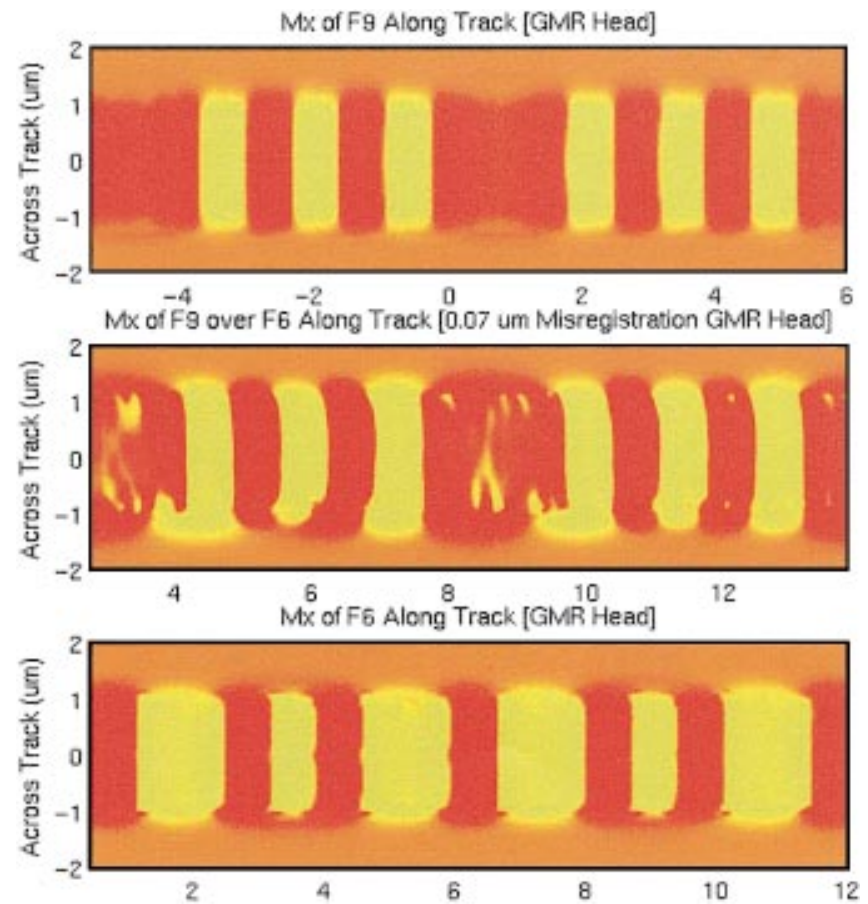

FIG. 4. (Color) Reconstructed image of F6 overwritten by F9: $0.07 \mu \mathrm{m}$ misregistration. 
MFM Of F9 Over F6: 0.3 um Misregistration
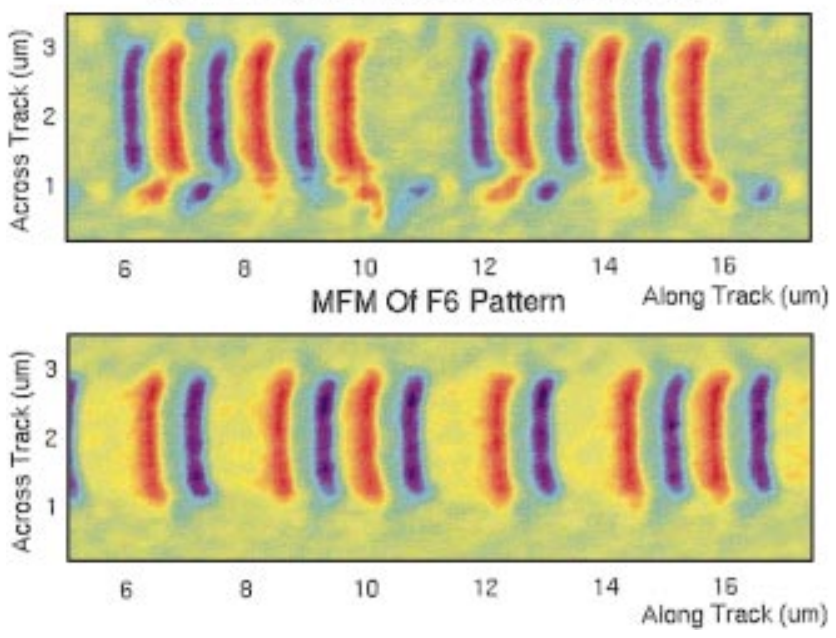

FIG. 5. (Color) MFM image of F6 overwritten by F9: $0.3 \mu \mathrm{m}$ misregistration.

track does not produce any magnetic field. This is because the magnetization within the track is divergence-free but not curl-free.

Thus $M_{x}$ and $M_{y}$ in formula (1) are meant to satisfy the additional equation:

$$
\operatorname{curl}_{z} \mathbf{M}=\frac{\partial M_{y}}{\partial x}-\frac{\partial M_{x}}{\partial y}=0 .
$$

Now, by using Eqs. (1)-(3), the measured response function $R$ of the MR reading element, and the Fourier transform technique, the curl-free component distribution of magnetization can be fully retrieved from the scalar raw image $S(x, y)$. The mathematical details of the reconstruction technique can be found in our previous work. ${ }^{2}$

The imaging technique described above has been implemented and extensively tested using a Guzik model 1701 MP spin-stand. The main emphasis has been on imaging of edge areas of tracks overwritten with small misregistrations ranging from 0.3 to $0.07 \mu \mathrm{m}$. In our experiments, giant magnetoresistive (GMR) heads (produced by ALPS) with write widths of $1.1 \mu \mathrm{m}$ and read widths of $0.7 \mu \mathrm{m}$ have been used. First, F6 patterns (hexadecimal F6 $=11110110$ in binary notation) were recorded and then they were overwritten by F9 patterns (hexadecimal F9 $=11111001$ in binary notation) with controlled misregistrations ranging from 0.3 to 0.07 $\mu \mathrm{m}$. The overwritten tracks were scanned and the collected raw images were reconstructed. Figures 2, 3, and 4 show the reconstructed images of F6 patterns overwritten by F9 patterns with misregistration of $0.15,0.09$, and $0.07 \mu \mathrm{m}$, respectively. These figures show only the $M_{x}$ (along-track) component of magnetizations. In order to emphasize the binary

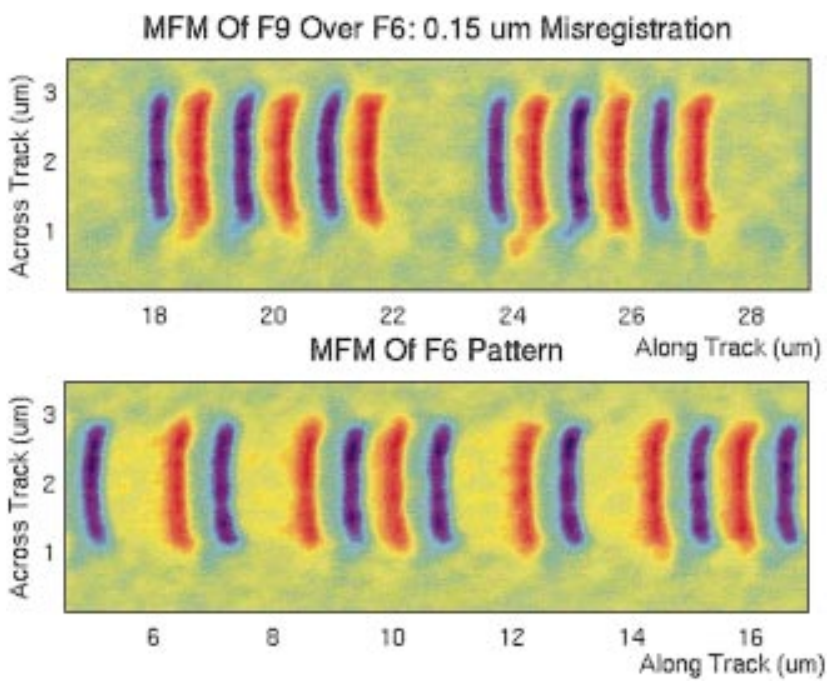

FIG. 6. (Color) MFM image of F6 overwritten by F9: $0.15 \mu \mathrm{m}$ misregistration.

nature of the patterns, the color contrast of the images of $M_{x}$ has been deliberately saturated. The artifact of this saturation is that noise has also been enhanced, as can be seen from Figs. 2, 3, and 4.

To assess the accuracy and the resolution of the described spin-stand imaging technique, extensive comparison of this technique with MFM imaging has been carried out. The sample results of this comparison are shown in Figs. 5 and 6 for misregistrations of 0.3 and $0.15 \mu \mathrm{m}$, respectively. Any such comparison, however, should be carried out in the context that MFM images represent magnetic charges of the patterns while the reconstructed spin-stand images are the magnetization distributions of the patterns. Still, it remains apparent from Fig. 6 that remnants of the overwritten F6 patterns are barely visible on the MFM image, while on the spin-stand image (see Fig. 2) these remnants are wellpronounced with many interesting details. This comparison suggests that the developed spin-stand imaging technique has at least the same level of resolution and accuracy as the MFM imaging technique and it is clearly superior to the latter as far as the rate and conditions of image acquisition are concerned.

${ }^{1}$ D. Rugar, H. J. Mamin, P. Guethner, S. E. Lambert, J. E. Stern, I. Fadyen, and T. Yogi, J. Appl. Phys. 68, 1169 (1990).

${ }^{2}$ I. D. Mayergoyz, C. Serpico, C. Krafft, and C. Tse, J. Appl. Phys. 87, 6824 (2000).

${ }^{3}$ R. Madabhushi, R. D. Gomez, E. R. Burke, and I. D. Mayergoyz, IEEE Trans. Magn. 32, 4147 (1996).

${ }^{4}$ I. A. Beardsley, IEEE Trans. Magn. 25, 671 (1989).

${ }^{5}$ I. D. Mayergoyz, A. A. Adly, R. D. Gomez, and E. R. Burke, J. Appl. Phys. 73, 5799 (1993). 